\title{
Linear and nonlinear stability of drift-tearing mode
}

\author{
Q. $\mathrm{Yu}$ \\ Max-Planck-Institut für Plasmaphysik, EURATOM Association, D-85748 Garching, Germany
}

The drift-tearing mode stability is studied numerically using the (reduced) two fluid equations. With a set of experimental data as input, the mode is found to be driven unstable by the electron temperature gradient when the classical perpendicular electron heat conductivity is taken into account. In the nonlinear phase two saturation regimes are found, a small magnetic island regime existing for an intermediate electron diamagnetic drift frequency and a large island regime for a sufficiently high bootstrap current fraction.

PACS: 52.35.Py, 52.35.Vd, 52.55.Tn, 52.35.-g 


\section{Introduction}

Tearing mode instabilities are of particular importance for magnetic fusion plasmas. In addition to the classical tearing mode driven by an unfavorable plasma current density gradient (a positive stability index $\Delta^{\prime}$ ), the perturbed bootstrap current drives the mode growth for a sufficiently high tokamak plasma pressure, leading to neoclassical tearing modes (NTMs), which have been found to limit the plasma pressure or even to cause disruptions in tokamak experiments [1-5].

Many NTMs observed in the experiments are triggered by additional perturbations like sawteeth or edge localized modes [3-5]. This phenomenon agrees with previous theories. Tearing modes are predicted to be stabilized by the electron diamagnetic drift in their linear phase even for a positive $\Delta^{\prime}$ if $\Delta^{\prime}<\left(\beta / \rho_{\mathrm{s}}\right)\left(\mathrm{L}_{\mathrm{s}} / \mathrm{L}_{\mathrm{Te}}\right)^{2}$, where $\rho_{\mathrm{s}}$ is the ion Larmor radius using the electron temperature, and $\mathrm{L}_{\mathrm{s}}$ and $\mathrm{L}_{\mathrm{Te}}$ are the scale length of the magnetic shear and electron temperature gradient, respectively [6]. The experimental values of $\Delta^{\prime}$ are usually found to be negative for tearing modes with poloidal mode number $m \geq 3$. In the nonlinear phase the diamagnetic drift effect leads to the ion polarization current model of the threshold for NTM onset, which has attracted extensive studies [7-15]. In addition, the Glasser effect also provides a somewhat smaller stabilizing effect $[5,16,17]$.

In some tokamak discharges, however, NTMs grow spontaneously [18-20], indicating that tearing modes can also be linearly unstable. It was recently found that tearing modes can be either stabilized or destabilized by the electron temperature gradient, depending on the values of the electron diamagnetic drift frequency $\omega_{* e}$ and the electron heat conductivity [21].

Based on Ref. [21], a consistent numerical modelling of the drift-tearing mode is presented here, using two fluid equations and including both the parallel and the perpendicular transport, which allows to take into account both the bootstrap and the ion polarization current perturbations. With a set of the experimental data as input, the tearing mode is found to be 
driven unstable by the electron temperature gradient, providing a possible explanation for the spontaneous growing tearing mode observed in the experiments [18]. In the nonlinear phase two saturation regimes are found, a small island regime driven by the electron temperature gradient and a large island regime (NTM regime) driven by the perturbed bootstrap current.

\section{MODEL}

The large aspect-ratio tokamak approximation is utilized here. The magnetic field is defined as $\mathbf{B}=\mathrm{B}_{0 \mathrm{t}}-(\mathrm{kr} / \mathrm{m}) \mathrm{B}_{0 \mathrm{t}} \mathbf{e}_{\theta}+\nabla \psi \times \mathbf{e}_{\mathrm{t}}$, where $\psi$ is the helical flux function, $\mathrm{m} / \mathrm{r}$ and $\mathrm{k}=\mathrm{n} / \mathrm{R}$ are the wave vectors in $\mathbf{e}_{\theta}$ (poloidal) and $\mathbf{e}_{\mathrm{t}}$ (toroidal) direction, respectively, $\mathrm{R}$ is the major radius, and the subscript 0 denotes an equilibrium quantity. The ion velocity $\mathbf{v}_{\mathbf{V}} \mathrm{v}_{\|} \mathbf{e}_{\|}+\mathbf{v}_{\perp}$, where $\mathrm{v}_{\|}$and $\mathbf{v}_{\perp}=\nabla \phi \times \mathbf{e}_{\mathrm{t}}$ are the parallel (to the magnetic field) and the perpendicular velocity, respectively. The cold ion assumption is made as in previous papers $[6,7,21]$.

To obtain $\psi, v_{\|}, \mathbf{v}_{\perp}$, the electron density $n_{e}$ and temperature $T_{e}$, the electron continuity equation, the generalized Ohm's law, the equation of motion in the parallel and the perpendicular direction (after taking the operator $\mathbf{e}_{\mathrm{t}} \nabla \times$ ), and the electron energy transport equation, are solved [6-8,21]. Normalizing the length to the minor radius a, the time $t$ to $\tau_{\mathrm{R}}, \psi$ to $a B_{0 t}, \mathbf{v}$ to $a / \tau_{R}$, and $T_{e}$ and $n_{e}$ to their values at the magnetic axis, where a is the minor radius, and $\tau_{\mathrm{R}}=\mathrm{a}^{2} / \eta$ is the resistive time, these equations become

$$
\begin{aligned}
& \frac{d n_{e}}{d t}=d_{1} \nabla_{\|} j-\nabla_{\|}\left(n_{e} v_{\|}\right)+\nabla \cdot\left(D_{\perp} \nabla n_{e}\right)+S_{n}, \\
& \frac{d \psi}{d t}=E-\eta\left(j-j_{b}\right)+\Omega\left[\nabla_{\|} n_{e}+1.17(1+\alpha) \nabla_{\|} T_{e}\right], \\
& \frac{d v_{\|}}{d t}=-C_{s}^{2} \nabla_{\|} P / n_{e}+\mu \nabla_{\perp}^{2} v_{\|}, \\
& \frac{d U}{d t}=-S^{2} \nabla_{\|} j+\mu \nabla_{\perp}^{2} U,
\end{aligned}
$$




$$
\frac{3}{2} n_{e} \frac{d T_{e}}{d t}=(1+\alpha) d_{1} \nabla_{\|} j-T_{e} n_{e} \nabla_{\|} v_{\|}+n_{e} \nabla \cdot\left(\chi_{\|} \nabla_{\|} T_{e}\right)+n_{e} \nabla \cdot\left(\chi_{\perp} \nabla_{\perp} T_{e}\right)+S_{p}
$$

where $\mathrm{d} / \mathrm{dt}=\partial / \partial \mathrm{t}+\mathbf{v}_{\perp} \cdot \nabla, \mathrm{j}=\nabla^{2} \psi-2 \mathrm{nB}_{0 \mathrm{t}} /(\mathrm{mR})$ and $\mathrm{j}_{\mathrm{b}}=(\mathrm{r} / \mathrm{R})^{1 / 2}\left(\mathrm{c}_{\mathrm{e}} \mathrm{n}_{\mathrm{e}} \mathrm{T}_{\mathrm{e}}{ }^{\prime}+\mathrm{c}_{\mathrm{n}} \mathrm{T}_{\mathrm{e}} \mathrm{n}_{\mathrm{e}}{ }^{\prime}\right) / \mathrm{B}_{\theta}$ are the plasma and the bootstrap current density along the $\mathbf{e}_{\mathrm{t}}$ direction, respectively, $\mathrm{B}_{\theta}$ is the poloidal magnetic field, $c_{e}=1.1, c_{n}=3.0$, and the prime denotes $d / d r . U=-\nabla_{\perp}^{2} \phi$ is the plasma vorticity, $\mu$ the plasma viscosity, $\chi$ the heat conductivity, and $\mathrm{D}$ the particle diffusivity. $\mathrm{P}=\mathrm{n}_{\mathrm{e}} \mathrm{T}_{\mathrm{e}}$, the subscripts $\|$ and $\perp$ denote the parallel and the perpendicular components, respectively, and $\alpha=0.71[6-8,21] . S_{n}, S_{p}$ and $\mathrm{E}$ are the particle and heat source and the equilibrium electric field, respectively.

The parameters in equations (1)-(5) are given by $\mathrm{d}_{1}=\omega_{\mathrm{ce}} / \nu_{\mathrm{e}}, \Omega=\beta_{\mathrm{e}} \mathrm{d}_{1}, \mathrm{C}_{\mathrm{s}}=\left[\mathrm{T}_{\mathrm{e}} / \mathrm{m}_{\mathrm{i}}\right]^{1 / 2} /\left(\mathrm{a} / \tau_{\mathrm{R}}\right)$, and $S=\tau_{R} / \tau_{A}$, where $\beta_{\mathrm{e}}=4 \pi \mathrm{n}_{\mathrm{e}} \mathrm{T}_{\mathrm{e}} / \mathrm{B}_{0 \mathrm{t}}{ }^{2}, \omega_{\mathrm{ce}}$ and $\nu_{\mathrm{e}}$ are the electron cyclotron and the collisional frequency, and $\tau_{\mathrm{A}}=\mathrm{a} / \mathrm{v}_{\mathrm{A}}$ is the toroidal Alfven time. The drift-tearing mode stability is determined by these parameters and the transport coefficients for a given equilibrium. Equations (1)-(5) have been used to study the tearing mode stability before, but the perpendicular transport has been neglected [6-8].

In equation (5) $\chi_{\|}=\chi_{\| c}\left[1+\left(3.16 \mathrm{v}_{\mathrm{Te}} \mathrm{k}_{\|} / v_{\mathrm{e}}\right)^{2}\right]^{1 / 2}$ is used, following previous theories [21,22], where $\chi_{\| \mathrm{c}}=3.16\left(\mathrm{v}_{\mathrm{Te}}\right)^{2} / v_{\mathrm{e}}$ is the classical parallel electron heat conductivity, $\mathrm{k}_{\|}=\mathbf{B}_{0} \cdot \mathbf{k} /\left|\mathbf{B}_{0}\right|$, and $\mathrm{v}_{\mathrm{Te}}$ is the electron thermal velocity. $\chi_{\|}$reduces to $\chi_{\| c}$ in the limit $v_{\mathrm{e}}>>\mathrm{v}_{\mathrm{Te}} \mathrm{k}_{\|}$, and to $\mathrm{v}_{\mathrm{Te}} / \mathrm{k}_{\|}$in the collisionless limit.

\section{Numerical results}

Equations (1)-(5) are solved simultaneously using the initial value code TM1, which has been used for modelling NTMs and drift tearing modes before [21,23,24]. New numerical methods are utilized in the code to keep the numerical error at a very low level even for high values of $S$ and $\chi_{\Perp} / \chi_{\perp}[24-26]$. 


\subsection{Linear results}

An example for a spontaneously growing $\mathrm{m} / \mathrm{n}=3 / 2$ tearing mode on ASDEX-Upgrade has been shown in Ref. [18]. The corresponding deuterium plasma has the following parameters, $\mathrm{T}_{\mathrm{e}}=3.25 \mathrm{keV}, \mathrm{n}_{\mathrm{e}}=1.15 \times 10^{20} \mathrm{~m}^{-3}$, and $\mathrm{B}_{0 \mathrm{t}}=2 \mathrm{~T}$, leading to $\mathrm{S}=4.27 \times 10^{8}, \mathrm{~d}_{1}=2.7 \times 10^{7}$, $\Omega=8.45 \times 10^{5}, C_{s}=1.01 \times 10^{8}$, and $\chi_{\| c}=1.11 \times 10^{13} \mathrm{a}^{2} / \tau_{\mathrm{R}}$. The safety factor (q) profile is monotonic with a negative value of $\Delta^{\prime}$ for the $3 / 2$ mode, and the $3 / 2$ mode is therefore stable if the two fluids effects are neglected by taking $\Omega=\mathrm{d}_{1}=\mathrm{C}_{\mathrm{s}}=0$. The equilibrium electron temperature gradient length, $\mathrm{L}_{\mathrm{Te}}=\mathrm{T}_{\mathrm{e}} /(\mathrm{dT} / \mathrm{dr})$, equals $0.541 \mathrm{a}$ at the $\mathrm{q}=3 / 2$ rational surface. The local equilibrium density gradient is nearly zero. The collisional parameter $C=\left(\Delta_{\mathrm{D}} / \rho_{\mathrm{s}}\right)^{2}$ equals 0.0089, indicating the semi-collisional regime at $\mathrm{C}<<1[6]$, where $\Delta_{\mathrm{D}}=\left(\omega *_{\mathrm{e}} / \chi_{\|}\right)^{1 / 2}\left(\mathrm{RL}_{\mathrm{q}} / \mathrm{n}\right)$, and $\mathrm{L}_{\mathrm{q}}=\mathrm{q} /(\mathrm{dq} / \mathrm{dr})$ [6]. The above parameters provide the input data for our linear calculations except mentioned elsewhere. For obtaining sufficient spatial resolution, the radial grid size is $10^{-4} \mathrm{a}$ $10^{-5} \mathrm{a}$ around the rational surface.

In figure 1 the normalized (to $\tau_{\mathrm{R}}^{-1}$ ) linear growth rate $\gamma$ of the $3 / 2$ mode is shown as a function of the parameter $\Omega$ by the solid curve for $D_{\perp}=\chi_{\perp}=0.3 \mathrm{a}^{2} / \tau_{R}$ and $\mu_{\perp}=18.3 \mathrm{a}^{2} / \tau_{R}$. The classical perpendicular electron heat conductivity, $\chi_{\perp}=4.7 v_{\mathrm{e}}\left(\mathrm{v}_{\mathrm{Te}} / \omega_{\mathrm{ce}}\right)^{2}$, leads to $\chi_{\perp}=0.3 \mathrm{a}^{2} / \tau_{\mathrm{R}}$. From equation (2) it is found that $\omega_{*_{e}}=\Omega \mathrm{m}\left[\mathrm{L}_{\mathrm{n}}^{-1}+1.17(1+\alpha) \mathrm{L}_{\mathrm{Te}}^{-1}\right] \mathrm{a}^{2} /\left(\mathrm{r}_{\mathrm{s}} \tau_{\mathrm{R}}\right)$, where $\mathrm{L}_{\mathrm{n}}=\mathrm{n}_{\mathrm{e}} /\left(\mathrm{dn}_{\mathrm{e}} / \mathrm{dr}\right), \mathrm{r}_{\mathrm{s}}$ is the minor radius at the rational surface. A larger $\Omega$ value corresponds to a larger $\omega *_{\mathrm{e}}$ or $\beta$ value. For a sufficiently small $\Omega$, the diamagnetic drift effect is of course not important, and $\gamma=-2.0 \times 10^{3} / \tau_{\mathrm{R}}$ for $\Omega=0$. For a sufficiently large $\Omega$, corresponding to a high mode frequency, the mode is also stable in agreement with previous theories that neglect the perpendicular transport [6]. For intermediate values of $\Omega, 5 \times 10^{4}<\Omega<10^{6}$, however, the mode becomes unstable. The experimental value $\Omega=8.45 \times 10^{5}$ lies in the unstable region, in agreement with the experimental result of a spontaneous growth of the $3 / 2$ mode. The dotted curve in figure 1 is obtained by taking $\mathrm{D}_{\perp}=\chi_{\perp}=0.1 \mathrm{a}^{2} / \tau_{\mathrm{R}}$. The unstable region is narrower as $\chi_{\perp}$ decreases. The dashed curve in 
figure 1 shows the growth rate of the $6 / 4$ mode, with same input parameters as those for the solid curve. The $6 / 4$ mode has nearly the same unstable region as the $3 / 2$ mode. Only in the small $\Omega$ region the $6 / 4$ mode is more stable than the $3 / 2$ mode, because the $\Delta^{\prime}$ value is more negative for the $6 / 4$ mode, and the diamagnetic drift is not important in this case.

For small magnetic islands, assuming that the electron energy transport is dominated by the parallel and the perpendicular transport, the electron temperature perturbation in the lowest order is given by $\mathrm{T}_{\mathrm{e} 1}=0.3\left(\mathrm{~W} / \mathrm{W}_{\mathrm{c}}\right)^{2} \mathrm{~T}_{\mathrm{e} 0}{ }^{\prime}\left(\mathrm{r}-\mathrm{r}_{\mathrm{s}}\right)$ near the rational surface $[26,27]$, where $\mathrm{W}$ is the island width, $\mathrm{W}_{\mathrm{c}}=\mathrm{r}_{\mathrm{s}}\left(\chi_{\perp} / \chi_{\| c}\right)^{1 / 4}\left(\varepsilon_{\mathrm{s}} \mathrm{s}_{\mathrm{s}} \mathrm{n} / 8\right)^{-1 / 2}, \mathrm{~s}=\mathrm{rq} / \mathrm{q}, \varepsilon=\mathrm{r} / \mathrm{R}$, and the subscript $\mathrm{s}$ refers to take value at $\mathrm{r}=\mathrm{r}_{\mathrm{s}}$. Linearizing equation (5) one finds that the perpendicular electron heat transport term is much smaller than the $\mathrm{dTe} / \mathrm{dt}$ term if the parameter

$$
\omega_{\perp} \equiv \omega \mathrm{W}_{\mathrm{c}}^{2} / \chi_{\perp}
$$

is much larger than one, where $\omega \sim \omega_{*}$ is the mode frequency. With our input values $s_{\mathrm{s}}=0.78$, $\mathrm{r}_{\mathrm{s}}=0.578 \mathrm{a}, \varepsilon_{\mathrm{s}}=0.193$, and the other parameters as mentioned above, we find $\mathrm{W}_{\mathrm{c}}=1.27 \times 10^{-3} \mathrm{a}$. The solid curve in figure 1 shows a decay of the mode for $\Omega \geq 10^{6}$, corresponding to a mode frequency $2.98 \times 10^{6} / \tau_{\mathrm{R}}$ or $\omega_{\perp}=103$. Therefore, the decay of the mode in the large $\Omega$ region is due to a sufficiently high mode frequency such that the perpendicular heat transport is not important. In this limit the mode becomes stable as predicted by the previous theories obtained by neglecting the perpendicular transport [6]. When $\omega_{\perp}$ is not too large or too small, the perpendicular heat transport leads to unstable modes [21].

In figure 2 the linear growth rate of the $3 / 2$ mode is shown as a function of the normalized (to $\mathrm{a}^{2} / \tau_{\mathrm{R}}$ ) perpendicular heat conductivity $\chi_{\perp}$ by the solid curve for $\Omega=8.45 \times 10^{5}$, $\mathrm{D}_{\perp}=\chi_{\perp}$ and $\mu_{\perp}=18.3 \mathrm{a}^{2} / \tau_{\mathrm{R}}$. For a small $\chi_{\perp}$ the mode is stable, which agrees with the previous theoretical results obtained in the $\chi_{\perp}=0$ limit [6]. When $\chi_{\perp}$ is closing to the classical electron heat conductivity, $\chi_{\perp}>0.2 \mathrm{a}^{2} / \tau_{\mathrm{R}}$, the mode becomes unstable. It is seen that even the small classical perpendicular heat conductivity significantly affects the mode stability. The dotted 
curve is obtained with $\mu_{\perp}=97 \mathrm{a}^{2} / \tau_{\mathrm{R}}$, with the other parameters being the same as for the solid curve. The smaller growth rate for a larger $\mu_{\perp}$ indicates the stabilizing role of plasma viscosity. For the parameters $\chi_{\perp}=0.1 \mathrm{a}^{2} / \tau_{\mathrm{R}}$ and $\mu_{\perp}=18.3 \mathrm{a}^{2} / \tau_{\mathrm{R}}$, the linear growth rate is shown as a function of the normalized (to $\mathrm{a}^{2} / \tau_{\mathrm{R}}$ ) $\mathrm{D}_{\perp}$ by the dashed curve in figure 2 . The growth rate $\gamma$ decreases for a larger $\mathrm{D}_{\perp}$, showing that the perpendicular particle transport is stabilizing.

The mode stability has also been studied by taking the perpendicular transport coefficients to be at the anomalous transport level, $\chi_{\perp}=\mu_{\perp}=150 \mathrm{a}^{2} / \tau_{\mathrm{R}}$ (corresponding to $\left.\chi_{\perp}=\mu_{\perp}=0.5 \mathrm{~m}^{2} / \mathrm{s}\right), \mathrm{D}_{\perp}=\chi_{\perp} / 6$ (solid curve) and $\mathrm{D}_{\perp}=\chi_{\perp} / 5$ (dotted), as shown in figure 3 . We assume that the anomalous viscosity and heat diffusivity are caused by other short wave length modes not included in our model. The mode is unstable in the intermediate $\Omega$ region and stable in the small or large $\Omega$ region, being similar to the case shown in figure 1 . A larger value of $D_{\perp}$ is stabilizing, as also indicated in figure 2 , and narrows the unstable region in $\Omega$.

The detailed linear results had been described in Ref. [21], in which radial profiles of perturbations and the dependence of the linear growth rate on plasma parameters were presented. The mode is found to be more unstable for a smaller $C_{s}$ or a larger $\chi_{\|}, S$ and $d_{1}$. The instability has tearing parity, but the "constant $\psi$ " approximation, being true for the classical resistive tearing mode, is not valid if the diamagnetic drift frequency is not too small. In this case the helical flux perturbation has a fast change across the singular layer [21]. This mode is driven unstable by the equilibrium electron temperature gradient and can be found only when both the parallel and the perpendicular heat diffusion are taken into account. The perpendicular heat diffusion is usually neglected in previous drift-tearing mode theories [6-8]. For a sufficiently small $\chi_{\perp}$, the mode is found to be stable in agreement with previous results of the drift-tearing mode obtained in the $\chi_{\perp}=0$ limit [6]. 


\subsection{Nonlinear results}

In order to look into the nonlinear evolution of the mode, much more Fourier components are required to ensure numerical convergence, especially for larger values of $\chi_{\|} / \chi_{\perp}$ and S [24-26], leading to much longer calculation time. Due to the computational limit, the nonlinear calculation results presented in this section are obtained with a less realistic set of input parameters. The linear results obtained with these input data are however found to be similar to those shown in Section 3.1 and are therefore not presented.

The nonlinear time evolution of the normalized island width, W/a, is shown in figure 4 , with $S=5 \times 10^{6}, d_{1}=1.0 \times 10^{5}, C_{s}=1.0 \times 10^{6} \mathrm{a} / \tau_{R}, \chi_{\|}=5.0 \times 10^{8} \mathrm{a}^{2} / \tau_{\mathrm{R}}, \chi_{\perp}=\mu_{\perp}=10 \mathrm{a}^{2} / \tau_{\mathrm{R}}$, and $\mathrm{D}_{\perp}=\chi_{\perp} / 5$. The other inputs are kept unchanged. It will be shown later that the value of $\omega_{\perp}$ covers about the same range as Fig. 1. When the bootstrap current density fraction at the rational surface, $\mathrm{f}_{\mathrm{b}} \equiv\left[\mathrm{j}_{\mathrm{b}} / \mathrm{j}\right] \mid\left(\mathrm{r}=\mathrm{r}_{\mathrm{s}}\right)$, is 0 (dotted curves), the island decays for $\Omega \leq 2 \times 10^{3}$ but grows and saturates for $\Omega=4 \times 10^{3}$ and $6 \times 10^{3}$. The local electron temperature gradient is decreased by the island, which in turn leads to the mode saturation. The normalized ion-sound Larmor radius, $\rho_{\mathrm{s}} / \mathrm{a}$, equals $2.8 \times 10^{-3}, 4.0 \times 10^{-3}$ and $4.9 \times 10^{-3}$ for $\Omega=2 \times 10^{3}, 4 \times 10^{3}$ and $6 \times 10^{3}$, respectively. For $\mathrm{f}_{\mathrm{b}}=0.1$ (solid curves), the mode further develops into the NTM and saturates at a larger amplitude for $\Omega=2 \times 10^{3}$ and $4 \times 10^{3}$. For $\Omega=6 \times 10^{3}$ the saturated island width becomes comparable to that obtained with $\mathrm{f}_{\mathrm{b}}=0$, indicating that the diamagnetic drift dominates the nonlinear saturation amplitude in this case.

In some parameter space the nonlinear saturation level is found to be affected by the initial island width $\mathrm{W}_{0}$ at $\mathrm{t}=0$ given by the input data. The time evolution of the normalized island width is shown in figure 5 for different values of $\mathrm{W}_{0}$, with $\mathrm{f}_{\mathrm{b}}=0.1, \Omega=6 \times 10^{3}$ and the other input parameters being the same as those for figure 4 . The value of $\rho_{\mathrm{s}} / \mathrm{a}$ equals $4.9 \times 10^{-3}$. Two nonlinear saturation regimes are seen. The island saturates at a low level for $\mathrm{W}_{0}<0.062 \mathrm{a}$ $\left(\mathrm{W}_{0}<1.7 \mathrm{~W}_{\mathrm{c}}\right)$ but develops into the NTM regime in the opposite limit. Such a threshold for the 
onset of NTMs is often seen in tokamak experiments [2-5].

Corresponding to figure 5, the time evolution of the normalized mode frequency is shown in figure 6. The mode frequency approaches a higher (lower) value for the smaller (larger) island case. The island drifts in the electron diamagnetic drift direction as expected [15]. The mode frequency and the diamagnetic drift frequency in the linear phase are shown by the horizontal dotted and dashed lines.

In figure 7 the corresponding local radial electron temperature profiles are shown. The solid and dotted curves are for the large and the small island case in figure 5, and the dashed curve shows the original equilibrium temperature profile. Due to the fast parallel transport in the island region, the local temperature profile is more flattening for a larger island [26,27], leading to a corresponding lower mode frequency than that of a smaller island as seen in figure 6. The smaller local temperature gradient results in a weaker effect of the diamagnetic drift, so that the mode develops into the NTM regime with a larger initial island width as shown in figure 5.

The normalized saturated island width is shown in figure 8 as a function of $\Omega$. The value of $\rho_{\mathrm{s}}$ /a changes from $2.8 \times 10^{-3}$ to $8.9 \times 10^{-3}$ with increasing $\Omega$ from $2 \times 10^{3}$ to $2 \times 10^{4}$, as $\rho_{\mathrm{s}} / \mathrm{a} \sim \Omega^{1 / 2}$ when other parameters are unchanged. The solid curve is obtained with $\mathrm{f}_{\mathrm{b}}=0$. In this case the island saturates at a lower amplitude of the order of $\mathrm{W}_{\mathrm{c}}\left(\mathrm{W}_{\mathrm{c}}=0.037 \mathrm{a}\right)$ for intermediate values of $\Omega\left(\omega_{*}\right)$. For $f_{b}=0.1$ and a small $W_{0}$ (dotted), the saturated island width are characterized by two regimes. For $\Omega \leq 4 \times 10^{3}$, the bootstrap current perturbation leads to large islands, corresponding to the NTM regime. For a higher $\Omega$, the island width is approximately the same as that obtained with $\mathrm{f}_{\mathrm{b}}=0$, showing the important role of the electron diamagnetic drift (ion polarization current) in determining the nonlinear saturation level for a sufficiently large $\Omega$. For $\mathrm{f}_{\mathrm{b}}=0.1$ and a large $\mathrm{W}_{0}$ (dashed), the NTM regime extends to a larger $\Omega$ value than that with a small $\mathrm{W}_{0}$. When $\Omega$ is high enough $\left(\Omega>2 \times 10^{4}\right)$, the island decays even for $\mathrm{f}_{\mathrm{b}}=0.1$. 
For more realistic input parameters, $S=1.4 \times 10^{7}, \mathrm{~d}_{1}=8.5 \times 10^{5}, \chi_{\| c}=7.4 \times 10^{9} \mathrm{a}^{2} / \tau_{\mathrm{R}}$, and $\chi_{\perp}=\mu_{\perp}=3.1 \mathrm{a}^{2} / \tau_{\mathrm{R}}$, the normalized saturated island width is shown in figure 9 as a function of $\Omega$ by the solid curve for $f_{b}=0$. The value of $\rho_{\mathrm{s}} /$ a changes from $4.2 \times 10^{-3}$ to $1.3 \times 10^{-2}$ with increasing $\Omega$ from $4 \times 10^{3}$ to $4 \times 10^{4}$. The dotted curve in figure 9 is the same as the solid curve in figure 8 , shown here for comparison. It is seen that for larger values of $S, d_{1}$ and $\chi_{\| c}$, the island width is smaller because of a smaller $\mathrm{W}_{\mathrm{c}}\left(\mathrm{W}_{\mathrm{c}}=0.014 \mathrm{a}\right.$ in this case $)$, and the island exists in a larger $\Omega$ regime. In terms of the normalized parameter $\omega_{\perp}$ defined by equation (6), the unstable region exists for $5.0<\omega_{\perp}<70$, while the unstable region extends over $5.2<\omega_{\perp}<103$ and $6.7<\omega_{\perp}<54$ for the solid curves in figures 1 and 8 , respectively. The lower limits of $\omega_{\perp}$ for the unstable region are about the same for these three cases. The upper limit is somewhat larger with higher input values of $S, d_{1}$ and $\chi_{\|}$.

Using the classical transport coefficients, one finds from equation (6) that

$$
\omega_{\perp}=2.1(\mathrm{~m} / \mathrm{n})\left(\mathrm{R} / \mathrm{r}_{\mathrm{s}}\right)\left(\mathrm{L}_{\mathrm{q}} / \mathrm{L}_{\mathrm{Te}}\right)\left[\mathrm{L}_{\mathrm{Te}} / \mathrm{L}_{\mathrm{n}}+1.17(1+\alpha)\right]
$$

For figure 1 the unstable region is in $5.2<\omega_{\perp}<103$, corresponding to

$$
1.2<(\mathrm{m} / \mathrm{n})\left(\mathrm{R} / \mathrm{r}_{\mathrm{s}}\right)\left(\mathrm{L}_{\mathrm{q}} / \mathrm{L}_{\mathrm{Te}}\right)<25
$$

for $\mathrm{L}_{\mathrm{n}} \gg \mathrm{L}_{\mathrm{Te}}$. Assuming $\mathrm{m} / \mathrm{n}=3 / 2$ and $\mathrm{L}_{\mathrm{q}}=\mathrm{r}_{\mathrm{s}},(8)$ is simplified to

$$
0.8<\mathrm{R} / \mathrm{L}_{\mathrm{Te}}<16.7
$$

suggesting that small islands with their saturation width to be about $\mathrm{W}_{\mathrm{c}}$ could exist in tokamak plasmas, if $\mathrm{L}_{\mathrm{Te}}$ is not too small or too large.

The time evolution of the normalized island width is shown in figure 10 for a constant $\Omega, \Omega=6 \times 10^{3}$, but with different bootstrap current density fractions at the rational surface: $f_{b}=0.1,0.14,0.15$ and 0.2. The other input data are the same as those for Figure 5. The island saturates at a lower amplitude and has about the same width for $f_{b}<0.15$. A larger $f_{b}(\geq 0.15)$ drives the island into the NTM regime. In this regime the saturated island width is larger for a higher $f_{b}$ as expected [24]. The island width oscillates in time for the case $f_{b}=0.14$, being just 
below the critical value of $\mathrm{f}_{\mathrm{b}}$ for transition to the NTM regime.

Above results show that, the island tends to develop into the large island (NTM) regime for a larger $\mathrm{f}_{\mathrm{b}}$, while for a larger value of $\Omega\left(\omega_{\perp}\right)$ the island tends to saturate at a small width or even to become stable, indicating the different role of the perturbed bootstrap current and the diamagnetic drift (ion polarization current) in determining the nonlinear saturation level.

When keeping the other input parameters unchanged, both $f_{b}$ and $\omega_{\perp}$ increase with increasing electron temperature gradient. In the following results the values of $f_{b}$ and $\omega_{\perp}$ are chosen for a constant

$$
\mathrm{c}_{\mathrm{b}} \equiv \mathrm{f}_{\mathrm{b}} / \omega_{\perp},
$$

with the other parameters being the same as in figure 8 . This allows us to see the competition between the effects from the perturbed bootstrap current and from the diamagnetic drift. Depending on experimental parameters, for ASDEX-U H-mode plasmas the value of $\mathrm{f}_{\mathrm{b}}$ is usually in the range of $0.1-0.3$, while $\omega_{\perp}$ approximately ranges from 10 to 100 according to equation (7), leading to the value of $c_{b}$ to be the range of $10^{-3}-3 \times 10^{-2}$.

The time evolution of the normalized island width is shown in figure 11 with $c_{b}=6.2 \times 10^{-3}$ for three cases: (1) $f_{b}=0.05 \quad\left(\omega_{\perp}=8.0, \rho_{s} / a=3.7 \times 10^{-3}\right), \quad(2) \quad f_{b}=0.15 \quad\left(\omega_{\perp}=24\right.$, $\left.\rho_{\mathrm{s}} / \mathrm{a}=6.0 \times 10^{-3}\right)$ and $(3) \mathrm{f}_{\mathrm{b}}=0.3\left(\omega_{\perp}=48, \rho_{\mathrm{s}} / \mathrm{a}=8.5 \times 10^{-3}\right)$, with the other input data being the same as those for figure 8 . The solid (dotted) curve is obtained with a small (large) $\mathrm{W}_{0}$. For case (1) with a small $\mathrm{f}_{\mathrm{b}}, \mathrm{f}_{\mathrm{b}}=0.05$, the island always saturates at a small width, no matter what value is taken for $\mathrm{W}_{0}$. For case (2) with $\mathrm{f}_{\mathrm{b}}=0.15$, the island saturates at a small width for a small $\mathrm{W}_{0}$ but at a large amplitude for a large $\mathrm{W}_{0}$. For case (3) with $\mathrm{f}_{\mathrm{b}}=0.3$ the island always develops into the NTM since $f_{b}$ is high enough.

With a smaller $c_{b}, c_{b}=4.7 \times 10^{-3}$, the time evolution of the normalized island width is shown in figure 12 with (1) $f_{b}=0.175\left(\omega_{\perp}=38 \rho_{s} / a=7.5 \times 10^{-3}\right)$ and (2) $f_{b}=0.225 \quad\left(\omega_{\perp}=48\right.$, $\left.\rho_{\mathrm{s}} / \mathrm{a}=8.5 \times 10^{-3}\right)$. For case (1) with $\mathrm{f}_{\mathrm{b}}=0.175$, the island fluctuates at a small width. For case (2) 
with $\mathrm{f}_{\mathrm{b}}=0.225$, the island also fluctuates at a small width for a small $\mathrm{W}_{0}$ but saturates at a large amplitude for a large $\mathrm{W}_{0}$.

In figure 13 the normalized saturated island width is shown as a function of $\Omega$. The corresponding value of $\omega_{\perp}$ is given by $\omega_{\perp}=2.67 \times 10^{-3} \Omega$. The value of $\rho_{\mathrm{s}} / \mathrm{a}$ changes from $2.8 \times 10^{-3}$ to $8.9 \times 10^{-3}$ with increasing $\Omega$ from $2 \times 10^{3}$ to $2 \times 10^{4}$. The curve with circles (squares) corresponds to $\mathrm{c}_{\mathrm{b}}=4.7 \times 10^{-3}\left(6.2 \times 10^{-3}\right)$. The solid (dotted) curves are obtained with a small (large) $\mathrm{W}_{0}$. With $\mathrm{c}_{\mathrm{b}}=4.7 \times 10^{-3}$, the NTM regime is seen only for a large $\mathrm{W}_{0}$ in the large $\Omega\left(\mathrm{f}_{\mathrm{b}}\right)$ region. While for a small $\mathrm{W}_{0}$ only the small island regime is found. For the case with $c_{b}=6.2 \times 10^{-3}$, with increasing $\Omega\left(f_{b}\right)$ the island first saturates at a small width and then increases to a large one once a certain value of $\Omega\left(f_{b}\right)$ is reached, corresponding to the onset of NTMs. A larger value of $\mathrm{W}_{0}$ extends the NTM regime to a smaller $\Omega\left(\mathrm{f}_{\mathrm{b}}\right)$ value. It is seen that the nonlinear saturation level of the drift-tearing mode is determined by three parameters: $\Omega\left(\omega_{\perp}\right)$, $\mathrm{f}_{\mathrm{b}}$ and $\mathrm{W}_{0}$.

\section{Discussion and summary}

It is shown in the present paper that, the drift-tearing mode stability depends on the parameter $\omega_{\perp}=\omega *_{\mathrm{e}} \mathrm{W}_{\mathrm{c}}^{2} / \chi_{\perp}$. When $\omega_{\perp}$ is not too small or too large, the equilibrium electron temperature gradient drives the mode to grow even for a negative value of $\Delta^{\prime}$ and zero bootstrap current. Using the experimental values as the input data, the mode is found to be unstable in agreement with the experimental observations [18]. Since the $6 / 4$ mode is found to have nearly the same unstable region in $\Omega$ as the $3 / 2$ mode, the parameter $\Delta^{\prime}$ is not very important in determining the linear mode stability for a sufficient high $\omega_{*_{\mathrm{e}}}$ value.

Our nonlinear results indicate that, the nonlinear growth of the drift-tearing mode is determined by three parameters: $\omega_{\perp}, \mathrm{f}_{\mathrm{b}}$ and $\mathrm{W}_{0}$. The ion polarization current resulting from the diamagnetic drift could play either a stabilizing or a destabilizing role, depending on the values 
of $\omega_{\perp}$. In the case of $\mathrm{f}_{\mathrm{b}}=0$, this current drives the island to saturate at a small width about $\mathrm{W}_{\mathrm{c}}$ for an intermediate value of $\omega_{\perp}$. While for a sufficiently high $\omega_{\perp}$, the mode is stabilized by the ion polarization current as seen in Figure 8 . In the case with a finite $f_{b}$, both the bootstrap current and the ion polarization current determine the island saturation level. As the bootstrap current perturbation is larger for a higher $\mathrm{f}_{\mathrm{b}}$, while the ion polarization current is weaker for a smaller $\omega_{\perp}$, a sufficiently high $f_{b}$ and low $\omega_{\perp}$ are favorable for the mode to saturate at a higher level (NTM regime). The drift-tearing mode can spontaneously grow into the NTM only for a sufficiently high value of $\mathrm{f}_{\mathrm{b}} / \omega_{*_{\mathrm{e}}}\left(\mathrm{f}_{\mathrm{b}} / \omega_{\perp}\right)$. The effect of $\mathrm{W}_{0}$ (initial perturbation amplitude) results from the change of the electron temperature gradient around the rational surface by the island. Due to the fast parallel transport in the island region, the local temperature profile is more flattening for a larger island (Figure 7), leading to a weaker effect of the diamagnetic drift (ion polarization current), so that the bootstrap current density perturbation could drive the mode into the NTM regime more easily for a larger value of $\mathrm{W}_{0}$, as shown in Figure 5 . The NTM regime is therefore extended for a large $\mathrm{W}_{0}$. It is seen that the nonlinear growth and saturation of the island are affected by the heat transport in the island region, as which changes the electron diamagnetic drift frequency (ion polarization current) in the nonlinear phase. Although these nonlinear results are obtained for the parameters being lower than existing tokamak experimental values, they have shown the different behaviors of NTMs observed in the experiments $[1-5,18-20]$. For higher input parameters like those for figures 1-3, one could expect from figure 9 that the saturated island width is smaller in the small island regime due to the smaller value of $\mathrm{W}_{\mathrm{c}}$.

In existing theories of the ion polarization current perturbation, the effect of the perpendicular electron heat diffusion is not considered, so that the effect of $\omega_{\perp}$ and the small island regime are not found there [5-15]. Another feature of our numerical results is that the mode frequency due to the diamagnetic drift is self-consistently calculated, and the island is found to rotate in the electron diamagnetic drift direction as expected [15]. It should be 
mentioned that the value of $\omega_{\perp}$ is subject to uncertainty due to the uncertainty in the perpendicular electron heat transport. When taking into account the anomalous perpendicular electron heat transport due to the electrostatic turbulence, Eq, (7) is modified to

$$
\omega_{\perp}=2.1 \chi_{0}(\mathrm{~m} / \mathrm{n})\left(\mathrm{R} / \mathrm{r}_{\mathrm{s}}\right)\left(\mathrm{L}_{\mathrm{q}} / \mathrm{L}_{\mathrm{Te}}\right)\left[\mathrm{L}_{\mathrm{Te}} / \mathrm{L}_{\mathrm{n}}+1.17(1+\alpha)\right] \text {, }
$$

where $\chi_{0}=\left(\chi_{\perp \mathrm{c}} / \chi_{\mathrm{a}}\right)^{1 / 2}$ is the ratio between the classical and the anomalous perpendicular electron heat diffusivity. When $\chi_{\mathrm{a}}>>\chi_{\perp \mathrm{c}}, \omega_{\perp}$ is smaller, and the mode can more easily develop into the NTM regim, especially for a higher $\beta$ plasma for which $f_{b}$ and $c_{b}$ are larger. A smaller local magnetic shear at the rational surface, however, leads to a lager $\omega_{\perp}$ and therefore tends to keep the mode in the small island regime.

The small island regime found here is different from the micro tearing mode[6,28,29]. The micro tearing mode results from the inclusion of the energy dependence of the Coulomb collision frequency using drift kinetic equation, but such an effect has not been included in the equations used here. The parameter regime of the unstable mode is also different from that of the micro tearing mode $[6,28,29]$. The conditions for the onset of the micro tearing mode are $\mathrm{d} \ln (\mathrm{Te}) / \mathrm{d} \ln (\mathrm{n})>0.3$ and $v_{\mathrm{e}} / \omega_{*}<3$ [28]. However, both the micro tearing mode and the mode studied here have the same energy source, the electron temperature gradient. The small island regime found here is also different from the work in Ref. [30], in which the $\mathrm{m}=1$ resistive kink mode is studied in the presence of the diamagnetic drift, but both the parallel and the perpendicular heat diffusion, being essential for our results, are neglected there.

Since the modes with high mode numbers can also be destabilized even for a classical electron heat diffusivity, they will change the local temperature gradient and contribute to the anomalous transport once they are unstable. In figure 14 the normalized (to $\tau_{R}$ ) linear growth rate and frequency are shown as a function of poloidal mode number with $D_{\perp}=\chi_{\perp}=1.6 a^{2} / \tau_{R}$ and $\mu_{\perp}=96.9 \mathrm{a}^{2} / \tau_{\mathrm{R}}$. The other input parameters are the same as those for Figure 1 , and $\rho_{\mathrm{s}} / \mathrm{r}=2.17 \times 10^{-3}$. It is seen that the unstable region of the mode extends to high $\mathrm{m}$ values $(\mathrm{m} \sim 100)$, being similar 
to other short wave length modes studied in Ref. [31]. Future nonlinear studies using realistic tokamak plasma parameters are needed for a better understanding.

In summary, the drift-tearing modes is investigated using two fluid equations under the large aspect-ratio and the cold ion assumptions. It is found that with experimental data as input, the drift-tearing modes are driven unstable by the electron temperature gradient when the classical perpendicular electron heat conductivity is taken into account, providing a possible explanation for the spontaneous growing tearing mode observed in the experiments. In the nonlinear stage two saturation regimes are found. For a lower $\omega *_{\mathrm{e}}$ and/or a high fraction of the bootstrap current density, the mode develops into NTMs. In the opposite limit the saturated island width is essentially the same as that obtained without the bootstrap current perturbation, and the diamagnetic drift dominates the nonlinear saturation. A larger initial perturbation extends the NTM regime to the region with a lower bootstrap current density fraction or a higher $\omega_{*}$. 


\section{Reference}

[1] Z. Chang, J. D. Callen, E. D. Fredrickson, et al., Phys. Rev. Letts, 74, 4663(1995).

[2] H. Zohm, G. Gantenbein, A. Gude, S. Günter et al., Phys. Plasmas 8, 2009(2001).

[3] S. Günter,A. Gude, M. Maraschek, S. Sesnic, et al., Phys. Rev. Letters, 87, 275001(2001).

[4] R. J. La Haye, L. L. Lao, E. J. Strait, and T. S. Taylor, Nucl. Fusion 37, 397(1997).

[5] O. Sauter, R.J. La Haye, Z. Chang, D.A. Gates et al., Phys. Plasmas 4, 1654(1997).

[6] J.F. Drake, T.M. Antonsen, A.B. Hassam, and N.T. Gladd, Phys. Fluids 26, 2509(1983).

[7] Bruce D. Scott, and A. B. Hassam, Phys. Fluids 30, 90(1987).

[8] A. I. Smolyakov, Plasma Phys. Control. Fusion, 35, 657(1993).

[9] H. R. Wilson, J.W. Connor, R.J. Hastie, and C. C. Hegna, Phys. Plasmas 3, 248(1996).

[10] F. L. Waelbroeck and R. Fitzpatrick, Phys. Rev. Letts, 78, 1703(1997).

[11] F.L.Waelbroeck, J.W. Connor, and H. R. Wilson, Phys. Rev. Letters, 87, 215003(2001).

[12] J.W. Connor, F. L. Waelbroeck, and H. R. Wilson, Phys. Plasmas 8, 2835(2001).

[13] A.I. Smolyakov, H.R. Wilson, M. Ottaviani and F. Porcelli, Plasma Phys. Control. Fusion, 46, L1 (2004).

[14] R. Fitzpatrick, F. L. Waelbroeck, and F. Militello, Phys. Plasmas 13, 122507 (2006).

[15] F.L.Waelbroeck, Plasma Phys. Control. Fusion, 49, 905 (2007).

[16] A. H. Glasser, J. M. Greene, and J. L. Johnson, Phys. Fluids 19, 567(1976).

[17] H. Lütjens, J-F Luciani and X. F. Garbet, Phys. Plasmas 8, 4267(2001).

[18] A. Gude, S. Günter, S. Sesnic, ASDEX Upgrade Team, Nucl. Fusion 39, 127(1999).

[19] E. D. Fredrickson, Phys. Plasmas 9, 548(2002).

[20] R.J. Buttery, R. J. La Haye, P. Gohil, G.L. Jackson, et al, Phys. Plasmas 15 (2008) 056115.

[21] Q. Yu, S. Günter, B. D. Scott, Phys. Plasmas 10, 797(2003).

[22] Z. Chang and J.D. Callen, Phys. Fluids B4, 1167(1992). 
[23] Q. Yu, S. Günter, G. Giruzzi, K. Lackner, and M. Zabiego, Phys. Plasmas 7, 312(2000).

[24] Q. Yu, S. Günter and K. Lackner, Phys. Plasmas 11, 140(2004).

[25] S. Günter, Q. Yu, J. Krüger, and K. Lackner, J. Comp. Phys. 209, 354 (2005).

[26] Q. Yu, Phys. Plasmas 13 (2006) 062310.

[27] Richard Fitzpatrick, Phys. Plasmas 2, 825(1995).

[28] N. T. Gladd, J. F. Drake, C. L. Zhang, and C. S. Liu, Phys. Fluids 23, 1182(1980).

[29] A. B. Hassam, Phys. Fluids 23, 2493(1980).

[30] G. Ara, B. Basu, B. Coppi, G. Laval, M.N. Rosenbluth, and B.V. Waddell, Annals of Physics 112, 443 (1978).

[31] A.M. Dimits, G. Beteman, M.A. Beer, B.I. Cohen, et al, Phys. Plasmas 7, 969 (2000). 


\section{Caption}

Figure 1 The normalized (to $\tau_{\mathrm{R}}^{-1}$,) linear growth rate $\gamma$ versus $\Omega$. The solid (dotted) curve is for the $3 / 2$ mode with $D_{\perp}=\chi_{\perp}=0.3 a^{2} / \tau_{R}\left(0.1 a^{2} / \tau_{R}\right)$. The dashed curve is for the $6 / 4$ mode with same input parameters as those of the solid one.

Figure 2 The normalized linear growth rate $\gamma \tau_{R}$ of the $3 / 2$ mode versus the normalized (to $\left.\mathrm{a}^{2} / \tau_{\mathrm{R}}\right) \chi_{\perp}$ for $\Omega=8.45 \times 10^{5}, \mathrm{D}_{\perp}=\chi_{\perp}, \mu_{\perp}=18.3 \mathrm{a}^{2} / \tau_{\mathrm{R}}$ (solid curve) and $\mu_{\perp}=97.0 \mathrm{a}^{2} / \tau_{\mathrm{R}}$ (dotted). The dashed curve shows $\gamma$ versus the normalized (to $\mathrm{a}^{2} / \tau_{\mathrm{R}}$ ) $\mathrm{D}_{\perp}$ for $\chi_{\perp}=0.1 \mathrm{a}^{2} / \tau_{\mathrm{R}}$ and $\mu_{\perp}=18.3 \mathrm{a}^{2} / \tau_{\mathrm{R}}$.

Figure 3 The normalized (to $\tau_{\mathrm{R}}^{-1}$ ) linear growth rate $\gamma$ versus $\Omega$ for the $3 / 2$ mode with $\chi_{\perp}=\mu=150 \mathrm{a}^{2} / \tau_{\mathrm{R}}$. The solid (dotted) curve is for $\mathrm{D}_{\perp}=\chi_{\perp} / 6\left(\mathrm{D}_{\perp}=\chi_{\perp} / 5\right)$.

Figure 4 The nonlinear time evolution of the normalized island width, W/a, for $\Omega=2 \times 10^{3}$, $4 \times 10^{3}$ and $6 \times 10^{3}$, with $\mathrm{f}_{\mathrm{b}}=0.1$ ( solid curve) and $\mathrm{f}_{\mathrm{b}}=0$ (dotted).

Figure 5 The time evolution of the normalized island width with different initial island width $\mathrm{W}_{0}$ for $\mathrm{f}_{\mathrm{b}}=0.1$ and $\Omega=6 \times 10^{3}$. The island saturates at a low level for $\mathrm{W}_{0}<0.062 \mathrm{a}\left(=1.7 \mathrm{~W}_{\mathrm{c}}\right)$. In the opposite limit the island develops into the NTM.

Figure 6 Corresponding to figure 5, the time evolution of the normalized mode frequency. The mode frequency approaches a larger (smaller) value for the smaller (larger) island case. The island drifts in the electron diamagnetic drift direction. The mode frequency and the diamagnetic drift frequency in the linear phase are shown by the horizontal dotted and dashed lines.

Figure 7 The solid (dotted) curve is the local radial temperature profile for the large (small) island case at nonlinear saturation in figure 5, and the dashed curve is the original equilibrium temperature profile. The local temperature profile is more (less) flattening for a larger (smaller) island. 
Figure 8 The normalized saturated island width versus $\Omega$ for $\mathrm{f}_{\mathrm{b}}=0$ (solid curve) and for $\mathrm{f}_{\mathrm{b}}=0.1$ with a small (dotted) and a large $\mathrm{W}_{0}$ (dashed). The NTM regime extends to a larger $\Omega$ value with a large $\mathrm{W}_{0}$. When $\Omega$ is high enough $\left(\Omega>2 \times 10^{4}\right)$, the island decays even for $\mathrm{f}_{\mathrm{b}}=0.1$.

Figure 9 The normalized saturated island width versus $\Omega$ for $\mathrm{f}_{b}=0$. The solid curves is for $\mathrm{S}=1.4 \times 10^{7}, \mathrm{~d}_{1}=8.5 \times 10^{5}, \chi_{\| c}=7.4 \times 10^{9} \mathrm{a}^{2} / \tau_{\mathrm{R}}$, and $\chi_{\perp}=\mu_{\perp}=3.1 \mathrm{a}^{2} / \tau_{\mathrm{R}}$. The dotted curve is the same as the solid curve in figure 8 shown here fore comparison.

Figure 10 The time evolution of the normalized island width for $\Omega=6 \times 10^{3}$ with $f_{b}=0.1$, $0.14,0.15$ and 0.2 . A sufficiently large bootstrap current density fraction drives the saturated island from a low amplitude to a large one.

Figure 11 The time evolution of the normalized island width with $c_{b}=6.2 \times 10^{-3}$ for (1) $\mathrm{f}_{\mathrm{b}}=0.05\left(\omega_{\perp}=8.0\right),(2) \mathrm{f}_{\mathrm{b}}=0.15\left(\omega_{\perp}=24\right)$ and $(3) \mathrm{f}_{\mathrm{b}}=0.3\left(\omega_{\perp}=48\right)$. The solid (dotted) curve is obtained with a small (large) $\mathrm{W}_{0}$.

Figure 12 The time evolution of the normalized island width with $c_{b}=4.7 \times 10^{-3}$ for (1) $\mathrm{f}_{\mathrm{b}}=0.175\left(\omega_{\perp}=38\right)$ and $(2) \mathrm{f}_{\mathrm{b}}=0.225\left(\omega_{\perp}=48\right)$ starting from two different $\mathrm{W}_{0}$,

Figure 13 The normalized saturated island width versus $\Omega$. The corresponding value of $\omega_{\perp}$ is given by $\omega_{\perp}=2.67 \times 10^{-3} \Omega$. The solid (dotted) curve is obtained with a small (large) $\mathrm{W}_{0}$. The curve with circles (squares) corresponds to $c_{b}=4.7 \times 10^{-3}\left(6.2 \times 10^{-3}\right)$. The nonlinear saturation level is determined by three parameters: $\Omega\left(\omega_{\perp}\right), \mathrm{f}_{\mathrm{b}}$ and $\mathrm{W}_{0}$.

Figure 14 The normalized (to $\tau_{\mathrm{R}}$ ) linear growth rate and frequency as a function of poloidal mode number with $D_{\perp}=\chi_{\perp}=1.6 \mathrm{a}^{2} / \tau_{R}$ and $\mu_{\perp}=96.9 \mathrm{a}^{2} / \tau_{\mathrm{R}}$. The other input parameters are the same as those for Figure 1 , and $\rho_{\mathrm{s}} / \mathrm{r}=2.17 \times 10^{-3}$. The unstable region of the mode extends to high $\mathrm{m}$ values (m 100), being similar to other short wave length modes as shown in Ref. [31]. 


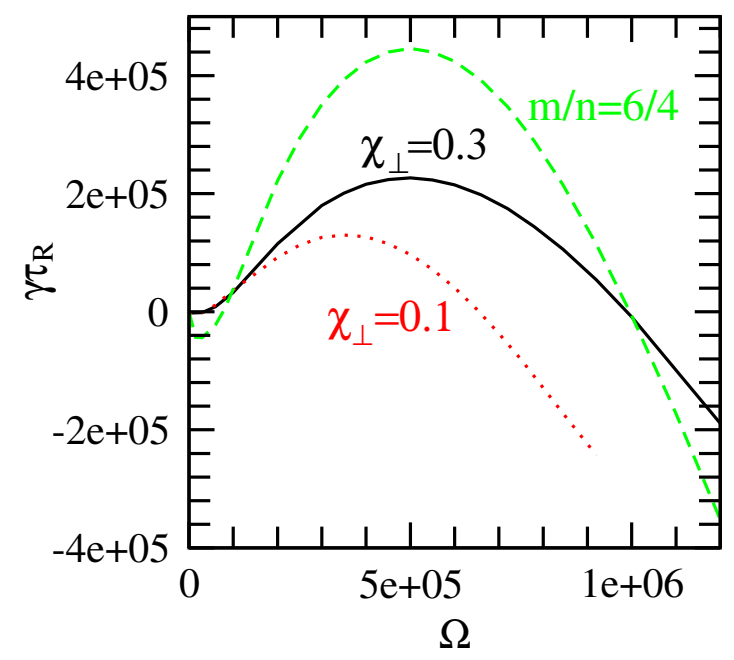

Figure 1 


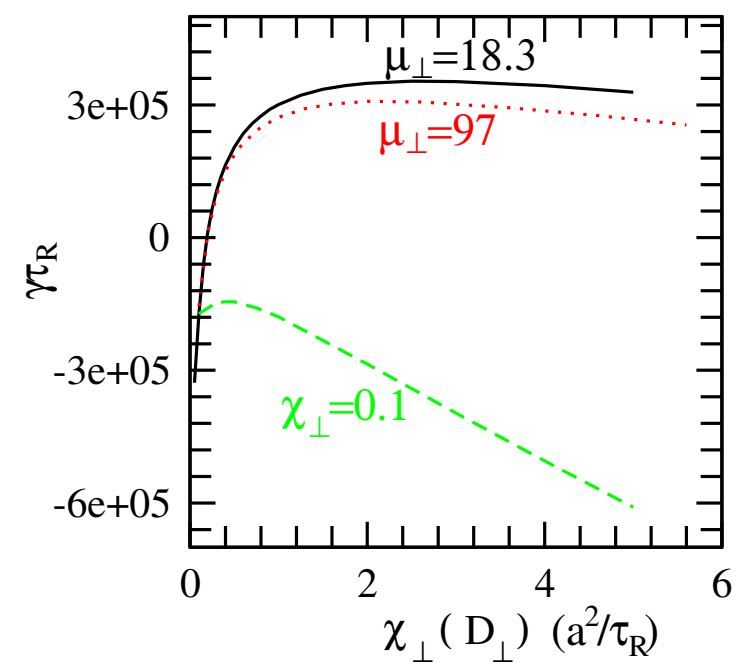

Figure 2 


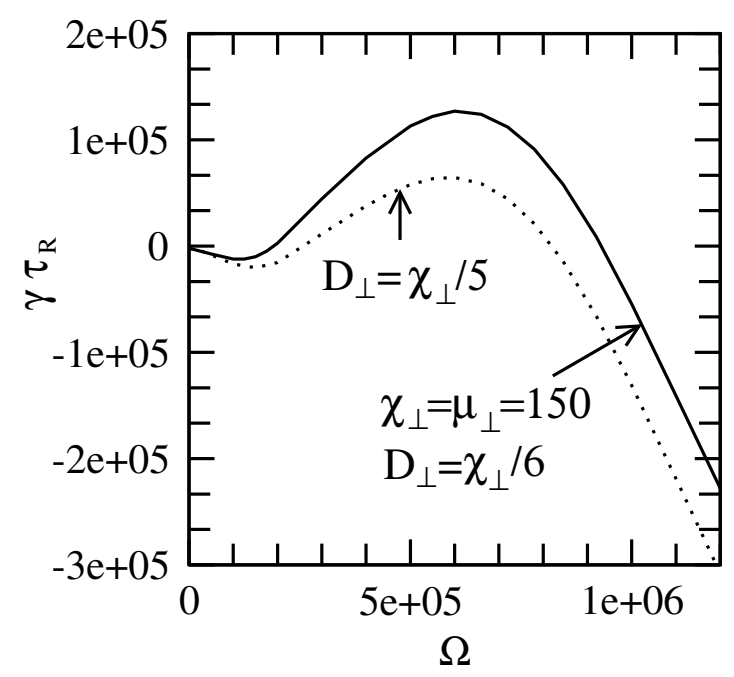

Figure 3 


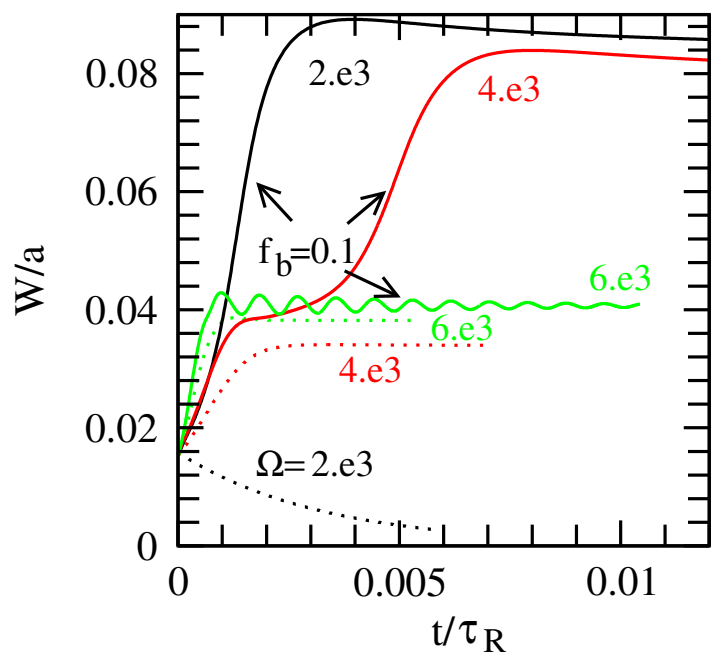

Figure 4 


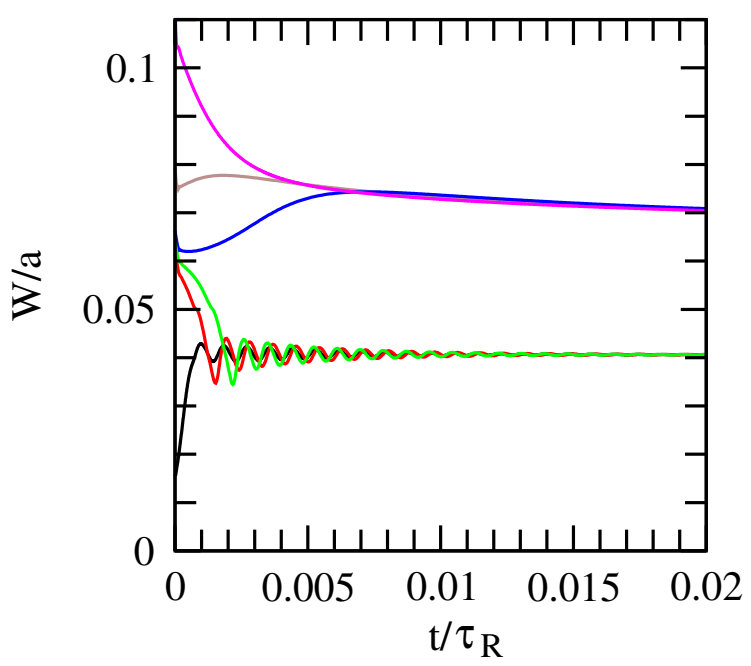

Figure 5 


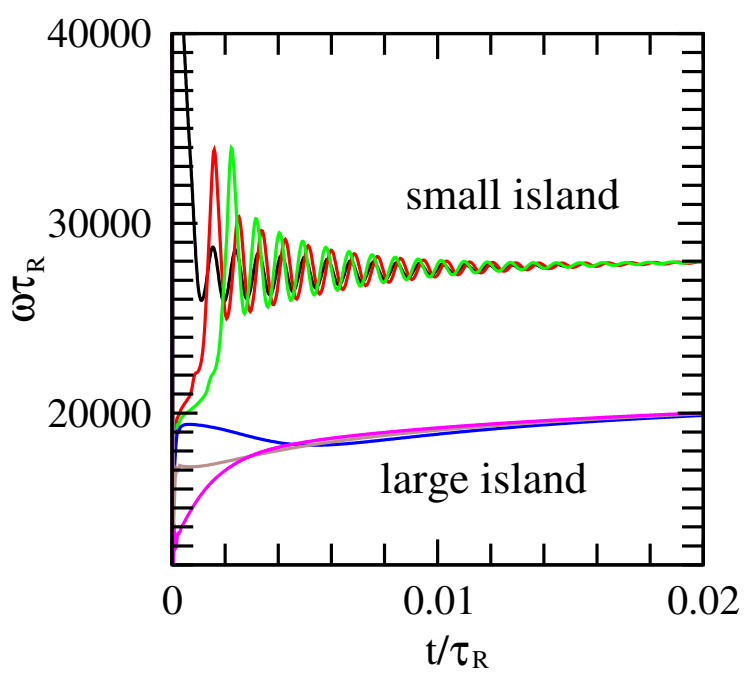

Figure 6 


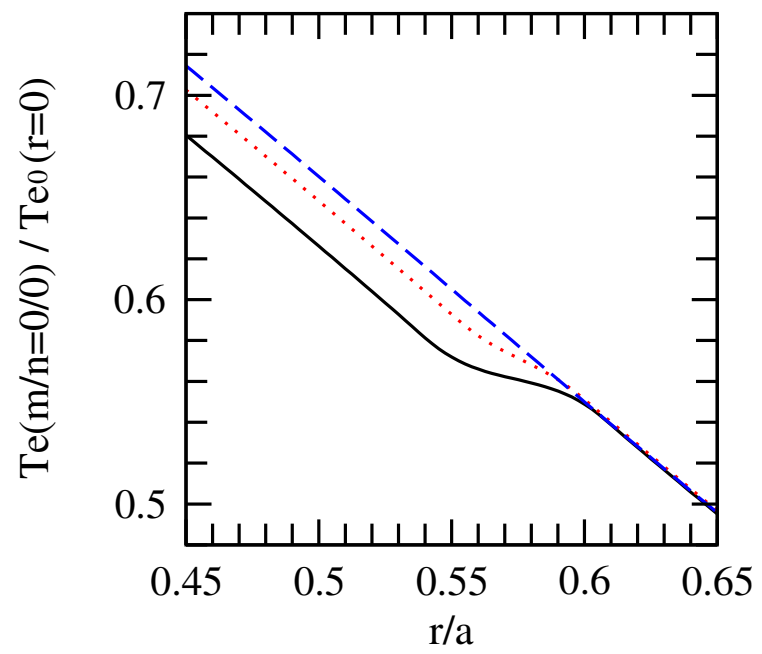

Figure 7 


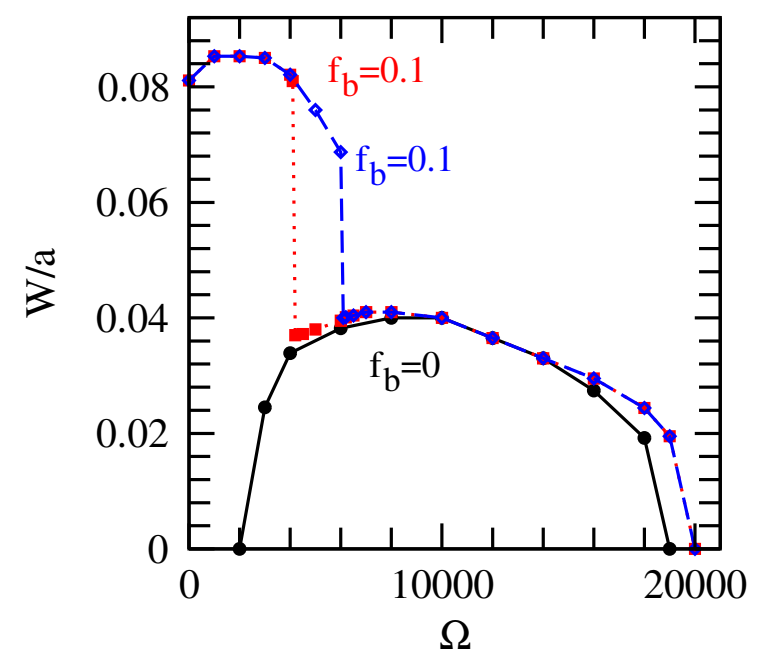

Figure 8 


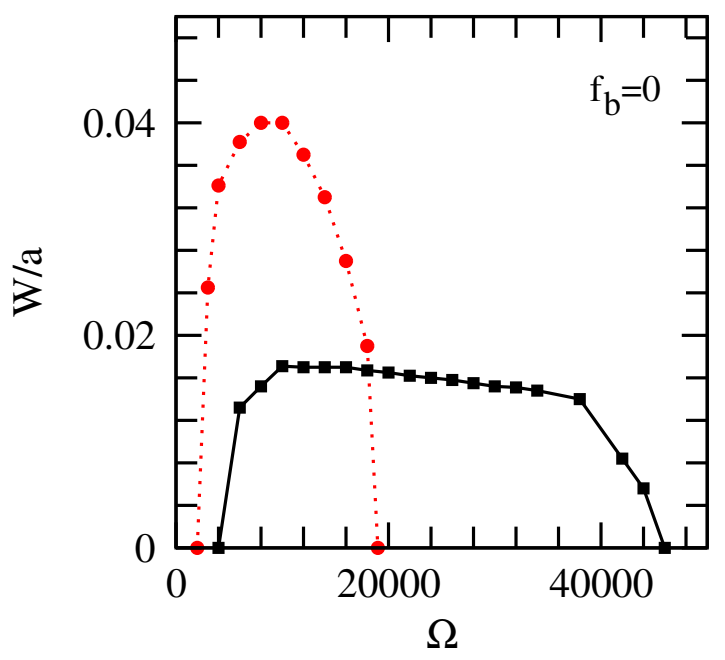

Figure 9 


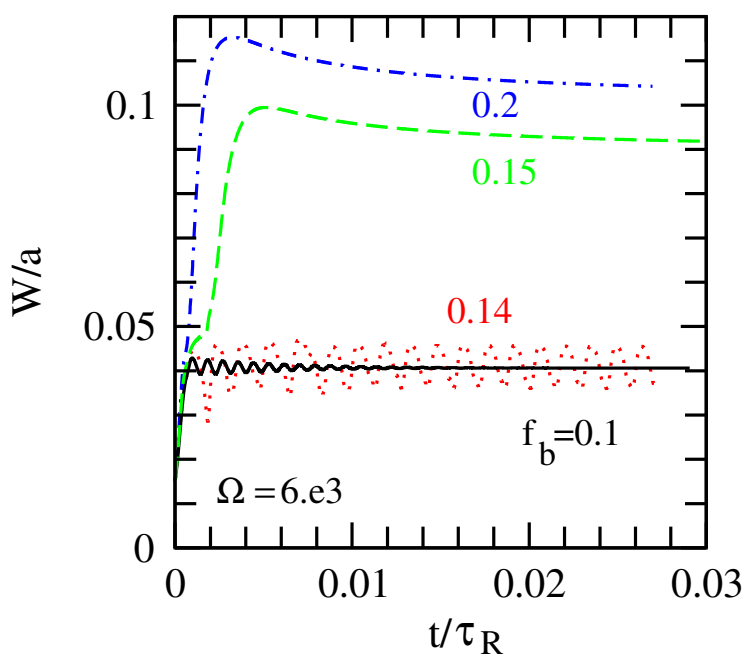

Figure 10 


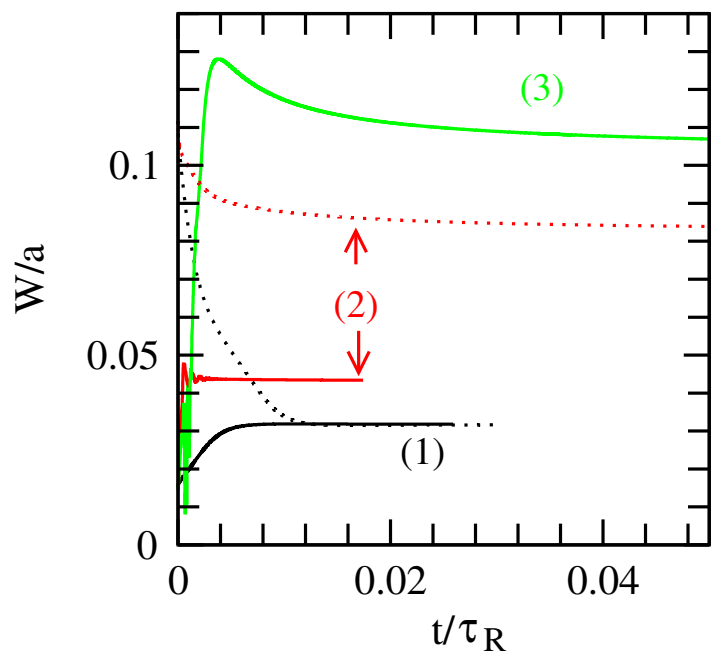

Figure 11 


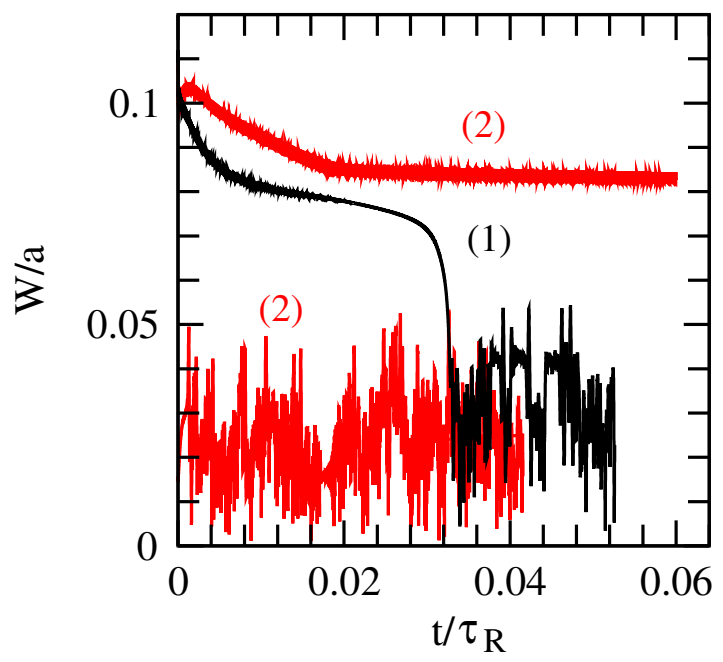

Figure 12 


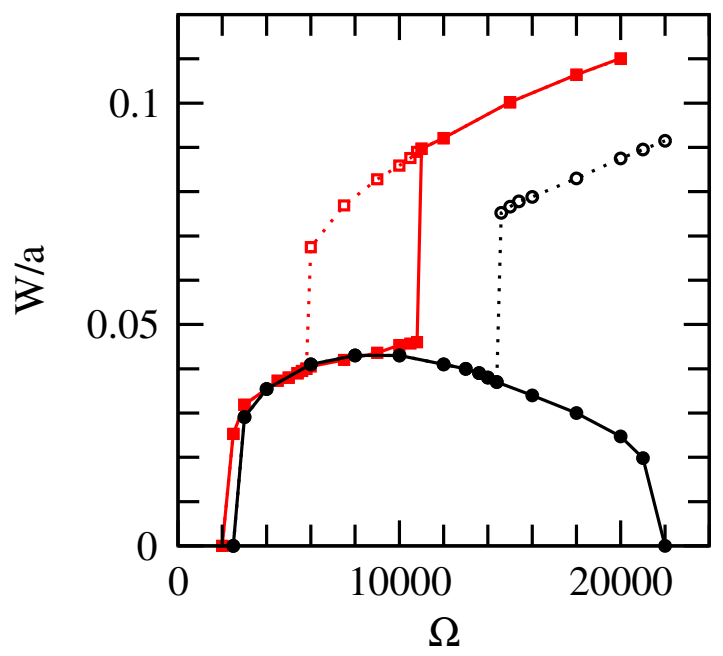

Figure 13 


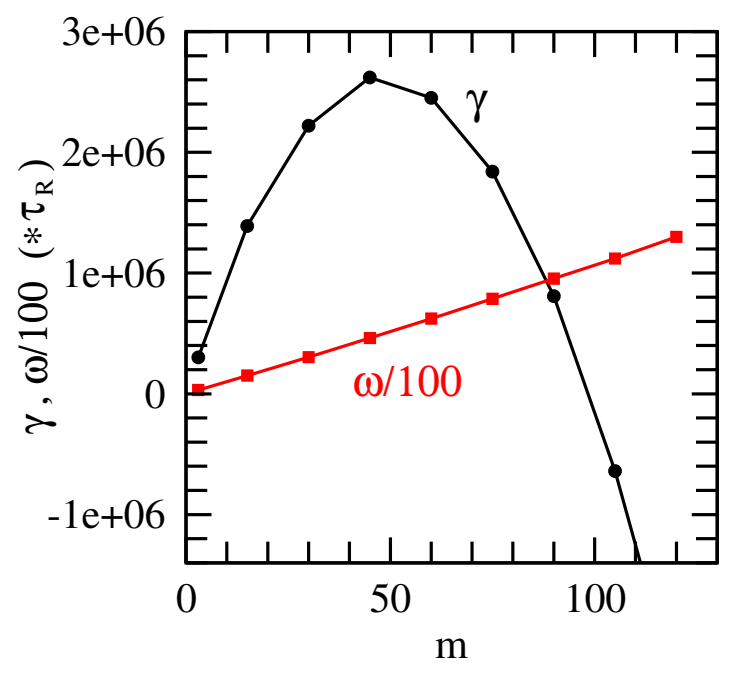

Figure 14 